\title{
CLIMAT ET VIOLENCE DANS LES ÉCOLES ÉLÉMENTAIRES TCHÈQUES : LA RELATION D'AUTORITÉ EN QUESTION DANS UN PAYS EN TRANSITION \\ Magdalena Kohout Diaz, Eric Debarbieux
}

Armand Colin | « Carrefours de l'éducation »

2010/1 n² 29 | pages 197 à 214

ISSN 1262-3490

Article disponible en ligne à l'adresse :

https://www.cairn.info/revue-carrefours-de-l-education-2010-1-page-197.htm

\section{Pour citer cet article :}

Magdalena Kohout Diaz, Eric Debarbieux« Climat et violence dans les écoles élémentaires tchèques : la relation d'autorité en question dans un pays en transition », Carrefours de l'éducation 2010/1 (n²9), p. 197-214.

DOI 10.3917/cdle.029.0197

Distribution électronique Cairn.info pour Armand Colin.

(C) Armand Colin. Tous droits réservés pour tous pays.

La reproduction ou représentation de cet article, notamment par photocopie, n'est autorisée que dans les limites des conditions générales d'utilisation du site ou, le cas échéant, des conditions générales de la licence souscrite par votre établissement. Toute autre reproduction ou représentation, en tout ou partie, sous quelque forme et de quelque manière que ce soit, est interdite sauf accord préalable et écrit de l'éditeur, en dehors des cas prévus par la législation en vigueur en France. Il est précisé que son stockage dans une base de données est également interdit. 


\title{
Climat et violence dans les écoles élémentaires tchèques: la relation d'autorité en question dans un pays en transition
}

\author{
A Magdalena Kohout-Diaz, Eric Debarbieux \\ mkohout@aquitaine.iufm.fr \\ debarberic@aol.com
}

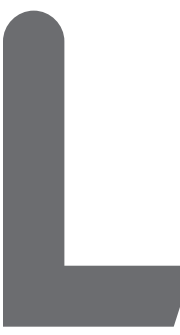

a violence à l'école est un sujet qui occupe la scène médiatique, politique, voire scientifique dans un grand nombre de pays (Debarbieux, 2006). Elle est officiellement à l'agenda des politiques éducatives européennes, qui prévoient à travers plusieurs programmes (Daphné par exemple) de prévenir le phénomène.

En République tchèque, pays sur lequel porte notre recherche, il est affirmé dans les milieux éducatifs et dans

la presse ${ }^{1}$ que le harcèlement entre pairs à l'école élémentaire a un caractère épidémique et dramatique (Havlínová, Kolár, 2001). Les relations entre élèves sont jugées catastrophiques. Leur qualité ainsi que les difficultés comportementales des enfants et des jeunes sont mises en avant de manière récurrente comme principal facteur explicatif d'une violence scolaire qui serait aigüe.

La recherche de climat scolaire et de victimation (Debarbieux, 2003; Kohout-Diaz, 2006) à laquelle nous nous référons a pour principal objectif de vérifier ces affirmations médiatiques, s'appuyant sur des travaux d'experts. L'enquête révèle en effet autre chose. La victimation subie par les élèves est relativement faible alors que la qualité des relations aux enseignants et autres adultes de l'école (les directeurs et leurs adjoints surtout) s'avère médiocre, se détachant sur le fond d'un climat scolaire global plutôt bon, voire exceptionnellement bon. Plus précisément, alors que l'autorité semble respectée selon les variables générales de l'enquête (faible recours aux punitions, félicitations fréquentes et abondantes, évaluations positives de l'école), parallèlement, les élèves déclarent ne pas estimer suffisamment leurs enseignants (peu de confiance en leurs compétences professionnelles, impolitesse).

1. Voir surtout le site de la prévention nationale du harcèlement scolaire [šikana] qui compile toutes les publications à ce sujet: http://sikana.org (30 janvier 2008). 
Si la récupération dite «experte » du thème de la violence à l'école peut être liée au souhait d'obtenir des financements nationaux ou européens ou encore de construire une réputation universitaire, il n'empêche qu'elle s'appuie sur un véritable malaise dans la fonction enseignante en République tchèque, malaise, présumons-le, lié aux mutations de la relation d'autorité dans une période historique marquée par la transition post-communiste qui a des effets en éducation et dans la socialisation professionnelles des enseignants.

Nous postulons que représenter l'autorité sur le plan éducatif, c'est exercer le pouvoir de se faire obéir (en y ayant été autorisé et/ou en s'y étant autorisé de soi même) pour, à terme, reconnaître l'élève comme auctor c'est-à-dire comme source de nouveauté, d'invention, ou éventuellement de critique et de rupture (Lacadée, 2003). C'est cette finalité qui seule la légitime. Dans un postcommunisme, la question des mutations des pratiques de l'autorité scolaire interroge donc à la fois les conceptions de l'autonomie et de la subjectivité des élèves et à la fois les reconfigurations des postures d'adhésion, d'adaptation ou de contournement des règles institutionnelles chez les professionnels de l'école en cours pendant la période communiste (Kott, Mespoulet, 2006).

Deux styles d'autorité se dégagent de notre enquête, soulignant l'évolution sociale des normes comportementales et des valeurs de l'école, croisée avec des effets de génération. Le rapport à l'autorité, exogène (s'exprimant sous la forme du respect de la politesse, de la correction, de la discipline et de l'absence de remise en question du discours du maître), semble mis à mal alors qu'un rapport d'autorité, endogène (sous la forme du respect de la singularité et de la différence, de la confiance, de la responsabilité, de l'éthique personnelle et professionnelle), paraît confus, émergent.

L'héritage de la «période autoritaire » rend difficile la réélaboration du rapport aux fonctions structurantes et éducatives de la loi (Devaux, 2006, p. 27). En effet, l'usage de la force et de la menace ainsi que la pratique généralisée de la corruption comme forme prévalente de l'échange (Kott, Mespoulet, 2006, p. 9) et l'omniprésence de l'idéologie marxiste (Prucha, 2007) semblent avoir marqué profondément les mentalités et entravé durablement l'exercice de la liberté pédagogique et le déploiement d'une réflexion axiologique sur l'éducation (Spilková, 2005). Le rapport de la communauté scolaire à la loi demeure ambigu, partagé entre la volonté de ne pas exercer de répression et la tentation de continuer l'économie des faveurs et des pratiques de contournement de la règle (Ragaru, 2006) dans le nouveau contexte démocratique et libéral. 


\section{Une recherche dans un pays postcommuniste: la République tchèque}

\section{Victimation et climat scolaire, une enquête internationale}

L'enquête réalisée en République Tchèque a été adaptée d'une enquête large menée au sein de l'Observatoire européen de la violence scolaire, qui a été initiée en France (Debarbieux, 1996), auprès d'échantillons divers, dont en 2003 un échantillon de 6268 élèves de 8 à 16 ans, en écoles primaires et au collège. Cette enquête a été menée dans une perspective comparative dans de nombreux autres pays, en Europe (Blaya, 2006) ou en Amérique du Sud et en Afrique (Debarbieux, 2006). C'est la première fois qu'un pays de l'ancien bloc de l'Est fait partie de cette enquête. En République tchèque nous avons interrogé, dans 15 établissements du premier et du second degré, 1638 élèves par questionnaires et 63 enseignants par entretien. Les quinze écoles se situent dans un milieu urbain et périurbain dans la région de Prague et d'Olomouc.

L'enquête par questionnaire vise à tester les victimations subies par les élèves, en tenant compte de leur caractère éventuellement répétitif. On sait en effet, et c'est la proximité de cette enquête avec celles sur le School Bullying (Olweus, 1993), que la victimation répétée peut avoir des conséquences importantes sur les individus, tant en termes de santé mentale que de réussite scolaire (voir par exemple Smith et al., 1999). Il s'agit d'une violence « ordinaire », certes moins spectaculaire que les violences paroxystiques, mais dont il ne faut négliger ni la prévalence, ni les conséquences (Hawker \& Boulton, 2000). Cette enquête est associée à l'évaluation du climat scolaire, comme dans d'autres enquêtes en particulier aux Etats-Unis (Gottfredson, 2001 ou Benbenishty \& Astor, 2006). Il est en effet largement prouvé combien la dégradation du climat scolaire et la victimation des élèves et des enseignants sont liées. Cette évaluation du climat scolaire est établie à l'aide de huit questions à échelles (alpha de Cronbach = 0,89 dans la version tchèque, ce qui est un excellent coefficient d'homogénéité). Ces questions portent sur l'évaluation globale de l'établissement, les interrelations entre élèves, avec les enseignants et personnels, l'environnement du quartier et la perception de la violence et de l'autorité. Plusieurs questions portent sur la perception de la justice scolaire et le mode disciplinaire employé. C'est à la partie de l'enquête sur le climat scolaire que nous allons nous intéresser dans cet article. La traduction des termes a posé quelques problèmes de compatibilité, nous y reviendrons plus loin.

\section{Une enquête en contexte: les caractéristiques essentielles du nouveau système éducatif tchèque.}

L'évolution du système éducatif tchèque se distingue au moins par trois traits significatifs. Le style pédagogique employé pendant la période totalitaire était 
directif, manifestant un irrespect de la personnalité de l'enfant, privilégiant un modèle d'enseignement "frontal » et la mémorisation de connaissances toutes faites imprégnées de la doctrine marxiste (Spilková, 2005, p. 26). L'exigence sociale de conformité, exacerbée, était peu compatible avec l'exercice de l'esprit critique et de l'autonomie des sujets (enseignants et élèves), inhérent à la citoyenneté démocratique. L'autorité des enseignants était essentiellement formelle, établie a priori par leur statut; "l'accent était mis sur la discipline extérieure, le conformisme » (ibid.)

Le nouveau contexte se caractérise par une autonomie accrue des établissements ${ }^{2}$ et une libéralisation générale du système éducatif (Prucha, 2007, p. 169). La forme passée de l'autorité statutaire des enseignants, fonctionnaires chargés de l'application de la politique d'État avant 1989 (Prucha, 2002), est de ce fait plutôt dévaluée, même si elle porte les traces de l'ambivalence du rejet tchèque à l'égard du communisme (Veuerník, 1994, Mayer, 2004, Kott, Mespoulet, 2006). La pédagogie de référence évolue, quant à elle, dans le sens d'une « humanisation [...], principe clé de la transformation de l'éducation nationale [tchèque] après $1989 »$. Une "pédagogie orientée vers l'enfant », qui opère un "retour à l'enfant », constitue une véritable nouveauté (Spilková, 2005). Le rapport des enseignants à la norme scolaire et la légitimation de leur pouvoir en appellent donc à une analyse: après avoir été les vecteurs de la normalisation, leur rôle dans la normativité à construire est central. Comment l'étayer?

Parallèlement, le système éducatif tchèque opère depuis 1989 une accentuation de la sélection à et par l'école (Greger, 2005, Prucha, 2007, p. 169). Le pouvoir sélectif des gymnasia (approx. lycées dans le système français, voir Prucha, 2007) est renforcé puisqu'il y a désormais trois périodes possibles (et non plus une) pour quitter l'école élémentaire et poursuivre la scolarité dans un gymnasium. Ces gymnasia constituent, comme sous la Première République (1918-1938), la voie privilégiée et prestigieuse pour accéder aux études supérieures, après sélection des élèves à fort capital socioéconomique et culturel, par un examen d'entrée. Ils dispensent un enseignement académique général et ont pour but principal de préparer l'entrée des élèves à l'Université. Leur expansion traduit le désir de retrouver intact un statu quo ante précédant la période communiste (Greger, 2005), un prestige traditionnellement associé à l'enseignement théorique, universitaire, académique (un prestige fondé sur la représentation d'une intelligence innée, naturelle), dévalorisé pendant la période communiste au profit de la dimension professionnelle de l'enseignement. Par effet rebond, cette sélection s'accroît aussi dans les écoles élémentaires (expansion des classes de renforcement, d'excellence [výberové trídy]), qui tentent de contrer le départ des élèves pour le gymnasium.

Or les donnés empiriques concernant l'état des pratiques pédagogiques sont insuffisantes, les résistances à mener des recherches sur ce thème (Prucha, 2002)

2. La décentralisation du système éducatif tchèque s'accentue de plus en plus depuis 1991, date des premières réformes. 
sont importantes et une « grande confusion terminologique [règne] dans la littérature pédagogique tchèque » (Spilková, 2005, p. 27). Le système éducatif tchèque évolue donc dans le sens d'une augmentation de l'injustice scolaire, abrasée en apparence par l'organisation éducative communiste, centralisée et strictement régulée dans le sens d'une réduction des inégalités et des différences sociales. Le refus de la différenciation pédagogique a en réalité abouti à une rigidité persistante des fonctionnements et au déni des particularités de chaque élève. L'autorité des enseignants et des adultes qui sont au moins les opérateurs impuissants de l'inéquité scolaire ainsi produite s'en trouve mise en cause.

\section{Les pratiques d'autorité en question, entre reproduction et émergence d'une critique de la part des élèves}

\section{De la violence entre pairs à la remise en question de l'autoritarisme des adultes: la qualité du lien pédagogique et le rapport à l'école}

La relation des élèves entre eux semble bien meilleure en République tchèque qu'en France. Seulement 11 \% des élèves tchèques jugent les relations entre pairs mauvaises ou très mauvaises contre $16 \%$ en France. En France, la cause de ces mauvaises relations est beaucoup plus liée aux problèmes de violence subies. En République tchèque par contre, la recherche montre abondamment combien la relation avec les enseignants paraît difficile, nous interrogeant sur les mutations de la représentation de l'autorité.

L'autorité, dans un premier sens, est un attribut (avoir de l'autorité) et consiste dans la dévolution à l'enseignant du pouvoir de (se) faire obéir. En ce sens, elle suppose donc une bonne qualité de la relation pédagogique, de la confiance et le respect des compétences professionnelles de l'expert reconnu (Reboul, 1989). Les enseignants de l'école élémentaire tchèque sont-ils en position d'expertise? L'enquête fournit deux types de réponses.

D’une part, les élèves tchèques sont bien moins nombreux que les français à faire état d'une une relation pédagogique de qualité (voir le tableau 1 ci-dessous). Comparativement aux français, ils perçoivent la qualité de l'enseignement comme moyenne voire mauvaise. 
Tableau I. - Évaluation de la qualité des relations aux enseignants, fr/cz.

\begin{tabular}{|c|c|c|c|c|}
\hline Profs Source & mauvaises & moyennes & bonnes & Total \\
\hline $\mathrm{Cz}$ & $18,6 \%$ (301) & $29,8 \%$ (483) & $51,7 \%$ (838) & $100 \%(1622)$ \\
\hline $\mathrm{Fr}$ & $15,0 \%(983)$ & $24,7 \%$ (1619) & $60,2 \%$ (3943) & $100 \%(6545)$ \\
\hline Total & $15,7 \%$ (1284) & $25,7 \%$ (2 102) & $58,5 \%$ (4781) & $100 \%$ (8167) \\
\hline
\end{tabular}

La dépendance est très significative. chi2 $=39,53$, ddl $=2,1-p=>99,99 \%$. \% de variance expliquée: 0,48 \%. Les cases encadrées sont celles pour lesquelles l'effectif réel est nettement supérieur (inférieur) à l'effectif théorique. Les valeurs du tableau sont les pourcentages en ligne établis sur 8167 citations.

D'autre part, le rapport à l'école (voir le tableau 2 ci-dessous) des élèves tchèques se caractérise par une incorrection/agressivité envers les enseignants; c'est ce qui spécifie le climat de l'école élémentaire tchèque, selon les élèves.

Pour mesurer ce rapport à l'école, nous avons procédé à une classification automatique des données, qui a permis de classer les élèves en quatre catégories, suivant leurs réponses aux questions sur le climat et la justice scolaires. Ces quatre catégories sont les suivantes: le groupe des élèves qui expriment une véritable joie de vivre à l'école («Tout Va Bien »); celui des élèves qui exprimant à la fois un refus des enseignants, des relations difficiles avec les autres élèves et un sentiment d'insécurité très fort ( Tout Va Mal»); les élèves qui pensent que le problème principal est l'agressivité vécue entre les enseignants («Aggprofs ») et enfin les élèves ni vraiment satisfaits, ni vraiment insatisfaits (« moyens »).

\section{Tableau 2. - Rapport à l'école, $F r / C z$}

\begin{tabular}{c|c|c|c|c|c|}
$\begin{array}{c}\text { Rapport à } \\
\text { l'école Source }\end{array}$ & TVM & Aggprofs & moyens & TVB & Total \\
\hline France & $13,8 \%(864)$ & $15,2 \%(954)$ & $28,6 \%(1794)$ & \multicolumn{1}{|c|}{$42,4 \%(1794)$} & $100 \%(6268)$ \\
\hline Cz & $17,3 \%(271)$ & $61,0 \%(953)$ & $5,6 \%(88)$ & $16,0 \%(250)$ & $100 \%(1562)$ \\
\hline Total & $14,5 \%(1135)$ & $24,4 \%(1907)$ & $24,0 \%(1882)$ & $37,1 \%(2906)$ & $100 \%(7830)$ \\
\hline
\end{tabular}

La dépendance est très significative. chi2 $=1596,66, \mathrm{ddl}=3,1-\mathrm{p}=>99,99 \% . \%$ de variance expliquée: 19,35\% Les valeurs du tableau sont les pourcentages en ligne établis sur 7830 citations.

Certes les élèves tchèques ne jugent pas majoritairement que « Tout Va $\mathrm{Mal}^{3}$ » mais ils sont, comparativement à la France, peu à déclarer que « Tout Va Bien » (TVB) pour pointer de manière très significative (61\%) l'agressivité de la relation aux enseignants, sensiblement plus dégradée qu'en France (15,2 \%).

Une relation d'autorité (endogène) dégradée est représentative, selon les élèves tchèques, de leur relation à leur école élémentaire. C'est l'une des composantes essentielles du climat scolaire dans le contexte donné. La construction de la

3. TVM; soulignons que cet item arrive tout de même en seconde position par sa fréquence. 
variable indiquant la nature spécifique du rapport à l'école (à partir de la synthèse des variables mesurant la qualité du climat) permet d'affirmer que la relation d'autorité, et non, par exemple, la qualité de la relation aux pairs, représente le climat de l'école élémentaire tchèque de façon la plus significative, comparativement à la France.

Plus exactement, ce que nous désignons ici par « autorité » s'exprime à travers l’impolitesse, l'incorrection [neslušnost] déclarée des élèves à l'égard de leurs enseignants, que nous avons comparée avec l'agressivité de la relation entre élèves et enseignants en France ${ }^{4}$. Ce choix méthodologique de traduire dans le questionnaire agressivité par impolitesse s'explique par la spécificité du contexte sociohistorique et linguistique tchèque: les termes étrangers et importés d'agrese/ agresivita [agression/agressivité] sont relativement inconnus, spécialisés, et surtout inadaptés et peu appliqués au contexte scolaire. Les acteurs du terrain évoquent plutôt l'impolitesse [neslušnost] ou même volontiers l'inconvenance [nevhodnost] pour qualifier les comportements scolaires (voir par exemple les travaux de Bendl sur l'autorité et la discipline, 2004).

Que désigne exactement cette «impolitesse » des élèves? Il est par exemple impoli de remettre en question la compétence de l'enseignant ou le fonctionnement du système éducatif, précisent les adultes interrogés au cours des entretiens. C'est être insolent [drzý]. Cette qualification est reproduite par les élèves. L'autonomie du jugement des élèves dans l'apprentissage, dans la construction des savoirs et l'autorisation de l'expression critique ne sont jamais posées comme fins éducatives alors que la nouvelle Loi sur l'éducation de 2004 en fait des axes majeurs des changements à entreprendre ${ }^{5}$.

Cette impolitesse a par ailleurs été définie par l'un de nos interlocuteurs enseignant comme le fait de «vouloir tout diriger» alors qu'il l'attribuait aux nouveaux parents. De là à définir la politesse comme obéissance, il n’y a qu'un pas. Être impoli, c'est ne pas obéir.

La relation d'autorité connaît donc une mutation sous les espèces d'une mise en question par les élèves de leur propre conformisme (politesse, convenances) scolaire, typique du style pédagogique autoritaire en vigueur sous la période communiste. Mais l'instauration de la part des élèves tchèques de cette valeur (impolitesse/agressivité) comme indicateur pertinent de leur propre rejet des enseignants traduit aussi paradoxalement l'adoption de leur part des normes conformistes des aînés. La catégorie des enseignants est reprise, adoptée pour dire le rejet de ces mêmes enseignants. Les résultats traduisent une évolution émergente et ambiguë des normes éducatives.

4. Dans le questionnaire français la question est: Y-a-t’il de l'agressivité entre élèves et professeurs? La version tchèque demande: Les élèves sont-ils corrects avec les professeurs?

5. Il s'agit du Programme cadre des contenus de l'enseignement fondamental. L'on peut voir sur ce point notamment le site du Conseil de l'Europe à propos du chapitre sur l'Education à la citoyenneté démocratique et aux droits de l'Homme: http://www.coe.int/t/f/coop \% C3 \% A9ration_culturelle/ education.e.c.d./Profiles_pays/(16.02.2006) 


\section{Les pratiques des enseignants en mutation: une mise en question par les élèves des compétences pédagogiques et des pratiques punitives héritées du communisme dans le nouveau contexte libéral}

L'autorité met également en jeu la reconnaissance par les élèves de la qualité de l'apprentissage (item « faire apprendre »). Or, comparativement aux élèves français, les élèves tchèques témoignent d'un doute à l'égard des compétences didactiques et pédagogiques de leurs enseignants (voir le tableau 3 ci-dessous), alors même que l'enquête se déroule dans des établissements plutôt favorisés socio-culturellement qui, dans leur majorité, collaborent étroitement et régulièrement avec l'Université pour la formation des enseignants. La qualité des apprentissages semble plutôt assez mauvaise.

Tableau 3. - Évaluation de la qualité de l'apprentissage, $\mathrm{Fr} / \mathrm{Cz}$

\begin{tabular}{|c|c|c|c|c|c|}
\hline $\begin{array}{l}\text { apprendre } \\
\text { Source }\end{array}$ & mal & assez mal & assez bien & bien & Total \\
\hline $\mathrm{Cz}$ & $2,7 \%$ (44) & $37,0 \%$ (597) & $42,8 \%$ (691) & $17,5 \%$ (283) & $100 \%(1615)$ \\
\hline $\mathrm{Fr}$ & $3,7 \%$ (238) & $18,9 \%(1232)$ & $23,3 \%(1516)$ & $54,1 \%(3522)$ & $100 \%(6508)$ \\
\hline Total & $3,5 \%$ (282) & $22,5 \%$ (1 829) & $27,2 \%$ (2207) & $46,8 \%$ (3805) & $100 \%(8123)$ \\
\hline
\end{tabular}

La dépendance est très significative. chi $2=741,02, \mathrm{ddl}=3,1-\mathrm{p}=>99,99 \%$. \% de variance expliquée: 8,98 \% Les cases encadrées sont celles pour lesquelles l'effectif réel est nettement supérieur (inférieur) à l'effectif théorique. Les valeurs du tableau sont les pourcentages en ligne établis sur 8123 citations.

En marge des questionnaires ou lors des questions ouvertes, les élèves déclarent d'ailleurs n'avoir d'estime, au fond, ni pour le savoir (inadapté, inutile, inintéressant), ni pour les pratiques pédagogiques des enseignants (rigides, peu interactives, ennuyeuses). Ils ne les considèrent pas réellement comme experts ni dans leur domaine pédagogique (relation éducative), ni didactique (maîtrise des savoirs spécifiques de leur discipline et de leur mise en œuvre). Ces résultats questionnent dans la mesure où ils contrastent fortement avec le climat globalement bon de l'école tchèque, révélé dans le reste du questionnaire (bonne évaluation générale, peu de violence, bonnes relations entre pairs). La dévaluation de la compétence de l'enseignant aux yeux des élèves ne produit donc pas une contestation violente et une désobéissance explicite à l'autorité adulte (autorité exogène). Une disjonction entre les apparences et les faits existe, qui témoigne du caractère émergent de l'expression critique chez les élèves mais aussi de leur difficulté à se détacher des normes éducatives de leurs aînés. Les élèves restent globalement disciplinés mais se déclarent impolis (exigence de conformité incorporée par les élèves et autono- 
mie du jugement interprétée comme impolitesse), sans respect pour l'expertise de leurs enseignants.

Les pratiques essentiellement répressives de l'autorité sous le régime communiste semblent donc avoir eu pour conséquence à l'école une suspension de la confiance des élèves dans les compétences éducatives et les savoirs de la génération précédente.

L'autorité en actes (faire autorité) interroge en effet aussi la pratique des sanctions et des félicitations à l'école. Elle est un acte d'affirmation de la loi. En ce sens, elle implique une représentation des sanctions, de leurs fonctions et leurs finalités, c'est-à-dire la formation à une pratique citoyenne active, autonome et démocratique à l'école. La sanction a pour fonction de structurer la subjectivité, de situer sa liberté dans un espace de droits, devoirs et interdits, d'organiser la vie collective. Elle a une fonction socialisante déterminante, constituant un socle de la pratique pédagogique (Prairat, 2001, 2003, 2005). Les enseignants tchèques font-ils autorité en ce sens? Deux réponses principales se dégagent.

Sous l'apparence d'un climat policé où la félicitation est très fréquente et la sanction rare, jugée comme relativement juste et respectée, se dévoile, du point de vue des enseignants, une plainte quant à la nouvelle impossibilité de punir et une mécompréhension de la valeur éducative de la sanction.

Du côté des élèves, le tableau 4 ci-dessous montre que, comparativement à la France, l'école tchèque punit peu. Le taux de félicitations reçues atteint 88,2 \%. En République tchèque. Les élèves déclarent être peu punis et souvent félicités. La relation éducative n'est pas autoritariste, si nous entendons par là des pratiques fréquentes et injustes (non acceptées, avec un recours éventuel à la force physique) de la punition.

\section{Tableau 4. - Punition, $\mathrm{Fr} / \mathrm{Cz}$}

\begin{tabular}{|c|c|c|c|}
\hline $\begin{array}{l}\text { punition } \\
\text { Source }\end{array}$ & oui & non & Total \\
\hline France 2003 & $52,4 \%$ (3418) & $47,6 \%$ (3104) & $100 \%(6522)$ \\
\hline République Tchèque & $40,0 \%(648)$ & $60,0 \%(974)$ & $100 \%(1622)$ \\
\hline Total & $49,9 \%$ (4 066) & $50,1 \%(4078)$ & $100 \%(8144)$ \\
\hline
\end{tabular}

La dépendance est très significative. chi2 $=80,62, \mathrm{ddl}=1,1-\mathrm{p}=>99,99 \%$. Odd-ratio : 1,66 \% de variance expliquée: 0,98 \% Les cases encadrées sont celles pour lesquelles l'effectif réel est nettement supérieur (inférieur) à l'effectif théorique. Les valeurs du tableau sont les pourcentages en ligne établis sur 8144 citations.

Cependant, la notation du comportement [známka z chování] subsiste, créant un malaise important. C'est la seule pratique scolaire qui ait été explicitement déclarée violente par les élèves. Apparaissant en réalité comme un moyen d'exclu- 
sion des élèves pour raisons ethniques et/ou socioéconomiques ou de handicap, elle fait fonctionner efficacement un processus de ségrégation alors que son efficacité éducative est qualifiée de nulle par tous les acteurs. Or son motif principal est l'impolitesse envers un adulte de l'école.

Du côté des enseignants, les explications avancées traduisent également un embarras profond. Ils se plaignent de ne " pas pouvoir sanctionner assez ", d'être muselés par la nouvelle mise en concurrence des établissements, de plus en plus autonomes (" cela ne plaît pas aux parents, alors si nous sanctionnons trop, ils vont changer l'enfant d'école »). Ils se disent également « lâchés » par l'institution (l'Inspection) qui, selon eux, soutient une " position radicale: il ne faut pas sanctionner car ce n'est pas éducatif ». L'idée avancée est que la démocratie, entendue à tort comme absence d'entraves, serait incompatible avec la sanction (confondue avec la punition).

Par conséquent, les enseignants ne s'autorisent pas à faire la loi parce qu'ils pensent que c'est réprimer. La sanction est envisagée essentiellement comme une répression/punition en vue d'obtenir l'obéissance et non comme facteur nécessaire d'éducation et de socialisation. L'enquête montre que sa nature est le plus souvent très formelle (remarques, remontrances, avertissements) et tous les acteurs signalent son inefficacité. De ce point de vue, les enseignants ne font donc pas véritablement autorité (même s'ils obtiennent l'obéissance) car ils ne parviennent pas à être perçus comme ceux qui contribuent à structurer le groupe social ou à préparer les élèves à le faire.

L'autorité est une fonction paradoxale qui «n'est pas due à la personne qui la manifeste, contrairement aux apparences » (Caumont, 2006): elle est reçue de l'autre. Elle implique un écart nécessaire entre ce qui est supposé par celui qui se soumet et l'idée qu'en a celui qui l'exerce ou la représente. Une assimilation de l'autorité à la répression (autoritarisme) joue sur l'illusion que cet écart peut/doit être réduit et masque la violence et l'agression. Dans le contexte du postcommunisme, l'autorité est profondément liée à la qualité du lien social et à son évolution au cours de la période totalitaire. Elle le révèle dans sa dimension de (perte de) confiance, de solidarité, de liberté et de respect civique de la loi, transcendante parce que commune. Nous voyons que l'autorité est relative aux processus de structuration subjective, qu'elle implique l'expression d'une autonomie critique, d'une articulation du nouveau et de l'ancien, une histoire personnelle et sociale assumée.

Mais la pratique de la sanction scolaire se dit ici soumise à la loi du marché libéral (Prokop, 2003, Spilková 2005, Prucha, 2007), ce qui augmente et occulte la difficulté à en réfléchir le sens. C'est comme si toute sanction était suspectée d'injustice en lien avec le régime passé, comme si elle était interdite dans un régime démocratique tel qu'il est imaginé (absence d'entraves) et comme si, en même temps, les pratiques anciennes, dont l'objectif premier est de sauver les apparences, persistaient (ambigüités, confusions sur les fins de l'éducation, manque de recul sur l'histoire ainsi qu'une coexistence paradoxale entre une 
fascination pour l'élitisme occidental, la sélectivité passée et la dénonciation de l'injustice sociale actuelle).

Quelles conséquences générales de ces résultats pour une redéfinition de l'autorité? L'enquête dément un préjugé facile selon lequel des comportements autoritaristes et violents généralisés, explicites, persisteraient à l'école tchèque et expliqueraient le rejet à l'égard des enseignants. Il s'avère cependant que l'autorité des enseignants et autres adultes de l'école ne repose pas sur une pratique signifiante de la sanction, en vue de permettre aux futurs citoyens de se construire de manière autonome, comme futurs membres actifs de la communauté politique. La persistance des notes de comportement ne peut, dans cette perspective, qu'être interrogée. Elle vise à éduquer un élève avant tout « poli » vis-à-vis des adultes mais éventuellement transgresseur à l'égard d'une loi qui demeure purement extérieure. La réticence à sanctionner que nous constatons témoigne d'une persistance de pratiques pédagogiques antérieures à 1989 et d'une responsabilité éducative insuffisamment réfléchie, associée à la précarisation du statut économique et socioprofessionnel des enseignants.

\section{La précarité des enseignants, signe d'une attitude sociale ambivalente à l'égard de leur statut}

L'autorité est en effet également liée à un statut (être une autorité) par lequel l'enseignant représente une loi transcendante (Arendt, 1968 et potestas, Prairat, 2001). L'évolution de la relation d'autorité est ce sens étroitement articulée aux changements politiques et sociaux qui se déroulent en République tchèque depuis 1989 (Parízek, 2000). Les enseignants tchèques sont-ils investis comme représentants de l'autorité?

$\mathrm{Au}$ cours de l'enquête, ils ne relèvent pas explicitement des problèmes de discipline mais évoquent les difficultés associées à l'évolution de leur statut très fréquemment. Ils le disent de plus en plus précaire du point de vue économique, se sentent peu reconnus par le champ social (parents surtout) ou par l'institution (par effet de la décentralisation) et démunis devant l'évolution des nouvelles normes sociales. Ils se plaignent de la dégradation des comportements, des mours, éventuellement dans le sens de nouvelles « pathologies sociales ${ }^{6}$ ». La question de l'autorité touche en fait pour eux plus à celle des nouveaux mobiles de l'obéissance qu'à la gestion d'une violence et une rébellion explicites de la part des élèves.

Mais c'est invariablement la précarisation économique (indéniable, Prucha, 2007) de leur métier qui est avancée comme cause première de toutes les autres difficultés et qui les oblige souvent à exercer un second métier, quelquefois sans

6. Le rapport national de la République tchèque pour la conférence de Stockholm signale déjà en 1999 la multiplication de comportements antisociaux chez les enfants.

Voir : http://www.social.coe.int/fr/cohesion/polfam/stockholm/Reptch.htm (08/04/2004). 
rapport avec leur qualification, ce qui les rend peu crédibles auprès des élèves et des parents:

«La situation économique des enseignants est difficile. C'est vrai que beaucoup d'entre eux sont obligés d'avoir un salaire d'appoint. Les femmes vendent souvent des produits cosmétiques sur leur lieu de travail. Ce sont souvent les produits Avon, vous connaissez peut-être? Elles peuvent aussi vendre des produits de nettoyage [...].»

Cette précarité économique, liée à la libéralisation de l'école et à l'accroissement du pouvoir du chef d'établissement, est aussi avancée comme l'explication principale d'une persistance du conformisme, à l'opposé de l'exercice des droits fondamentaux du citoyen comme le droit de grève, toujours massivement autocensuré. La faiblesse évoquée des syndicats (outil de contrôle du Parti, encastré dans les activités partisanes, plus que force de défense des intérêts professionnels) accentue le sentiment de solitude et le peu d'inclination à l'engagement social (Blaive, 2006) qui se disent, constituant une nouvelle mythologie de l'école contemporaine (Prokop, 2003):

« Nous avons théoriquement le droit de grève mais on ne l'exerce presque pas parce que chacun a peur de perdre sa place. Le Directeur, qui nous recrute, est chargé d'empêcher la grève. En plus, nous n'avons pas le soutien de l'opinion publique. Donc c'est pour rien. La dernière grève date des années quatre-vingt-dix. Et l'on s'est rendu compte que l'argent allait aux écoles non grévistes. Le syndicat était une association fictive [au cours de la période communiste], qui était plutôt chargée d'organiser des soirées. »

Cette situation socioéconomique contribue également à creuser des écarts importants avec certains élèves, de plus en plus nombreux, issus des familles aisées des "nouveaux entrepreneurs ${ }^{7}$ " La confrontation générationnelle se double d'une confrontation entre la persistance des valeurs et représentations sociales construites sous le communisme et celles qui sont associées à la démocratie libérale.

Pour faire face, les enseignants n'hésitent pas à faire des suppléances dans des disciplines dans lesquelles ils n'ont pas été formés. La pratique, illégale mais extrêmement répandue, contribue sans doute à expliquer partiellement la médiocre évaluation des pratiques didactiques par les élèves. C'est donc un des piliers de l'identité professionnelle des enseignants qui s'effrite puisque le métier était traditionnellement assez prestigieux du fait de la qualification universitaire assez élevée (niveau master) des enseignants (Prucha, 2007).

Le système éducatif décentralisé a, à leurs yeux, ôté tout pouvoir à l'inspection, qui n'a actuellement qu'une fonction consultative et de conseil, alors que les pouvoirs du chef d'établissement et des parents sont accrus. La qualité des

7. L'enquête se déroule dans quinze établissements où le niveau socioéconomique des familles est plutôt élevé. Il ne s'agit cependant pas d'établissements fréquentés par l'élite économique, comme le sont les gymnasium. 
enseignements est donc entièrement soumise à la loi de l'offre et de la demande malgré la loi de 2004 qui harmonise un programme national pour le baccalauréat. La sélection scolaire et en particulier l'exclusion des élèves Roms, handicapés et/ ou socio économiquement et/ou culturellement défavorisés est une pratique dont ils sont, bon gré mal gré, les agents. Leur attitude est partagée, ambivalente. Ils tiennent volontiers un discours d'impuissance et d'irresponsabilité, affirmant n'avoir pas le choix, associé à une fascination pour la sélection, valeur à la fois typique du glorieux passé de l'école tchèque et libérale, occidentale par excellence. Au cours des entretiens avec les enseignants, une seule posture professionnelle a été unanimement appréciée par les élèves. C'est celle d'un enseignant qui, utilisant pour ce les nouveaux fonctionnements scolaires, se positionne plutôt comme animateur et gestionnaire, organisant loisirs, voyages et activités extrascolaires avec les « dons de sponsors » récoltés pour la classe. Son autorité ne repose pas sur la représentation de la loi, de l'Etat et du savoir mais sur la possibilité qu'il offre de jouir des biens de consommation et des services. Celui dont les propos sont cités ci-dessous a par exemple, en plus de son activité d'enseignement, fondé sa propre école privée de musique 143 élèves dans les locaux-mêmes de l’institution (au sous-sol de l'école). En ce sens il a, dit-il, complètement « rompu avec l'esprit du communisme » :

« Je me suis débrouillé pour que nous collaborions aussi avec des assurances médicales c'est-à-dire que j'ai conseillé aux parents de prendre ces compagnies qui assurent des séjours de santé dans le coin, et alors ils nous font une réduction de 50 \% sur le séjour. Donc si nous partons deux fois par an, une fois en automne et une fois au printemps, les parents ne paient qu'un voyage en tout. "

La représentation qu'il donne de la classe est celle d'une entreprise autonome (qui « ne collabore plus trop avec l'école ») et libérale (" ce que les parents se dégottent, ce sera pour leurs enfants ») de loisirs éducatifs (voyages et autres « sorties ») où la solidarité et le souci d'équité deviennent incompréhensibles ${ }^{8}$. Au contraire, les dons des sponsors individuels et le profit immédiat, eux, sont largement encouragés. Il conclue que « ce que l'on donne pour tous, ça ne sert à rien »:

« Nous profitons des dons de sponsors pour la classe [pour payer ces voyages]. Nous avons 20 000,00 couronnes sur le livret de classe [666 euros environ]. Du coup, nous ne collaborons plus trop avec l'école; je donne le ton suivant: ce que les parents se dégottent, ce sera pour leurs enfants. Avant, avec cet argent, l'école achetait des manuels d'Anglais. C'est ce que je reprochais au Directeur: pourquoi on achète de telles choses avec cet argent et pourquoi ce n'est pas pour leurs propres enfants? Si j'ai un fils qui fait du sport à haut niveau, je ne vais pas non plus donner cet argent à toute léequipe... parce que si on le donne pour tous, ça ne sert à rien, l'argent se dilapide pour nombre de petites choses et on ne le voit nulle part. »

8. Ainsi, une autre enseignante de l'établissement expliquera que de ne pas fréquenter les élèves " à problèmes » de l'autre classe [problémové déti : il s'agit ici en réalité d'élèves moins performants] est une qualité qu'elle favorise chez ses élèves et qui relève d'une «intelligence naturelle » de la vie. 
Pour l'enseignant, consacrer de l'argent aux manuels d'anglais, supports d'apprentissage " pour tous », c'est le dilapider dans des « petites choses ». Il ne se situe plus comme représentant du savoir et pédagogue. Ce n'est pas en tant que tel qu'il cherche à être respecté. La construction des compétences civiques (solidarité, responsabilité collective, collaboration, esprit d'équipe, autonomie du jugement, normes qui font la légitimité du pouvoir exercé et de «la hiérarchie elle-même, dont chacun reconnaît la justesse », Arendt, 1968) qui légitime in fine l'autorité des adultes perd donc son sens.

Les enseignants, dans la spécificité de leur fonction sont donc socialement décrédibilisés, économiquement contraints et leur statut n’a pas été institutionnellement revalorisé. Lorganisation du nouveau système éducatif ne les aide pas à investir une autorité nouvelle, comme membres d'un nouveau « corps ». Leur statut est ambigu. Dans la société de transition, ils apparaissent à la fois comme les représentants privilégiés d'un passé avec lequel il faut rompre (agents de l'Etat) mais aussi paradoxalement comme les vecteurs du changement libéral (sélection, soumission à la loi de l'offre et de la demande) qui n'est pas toujours bien accueilli.

Ce malaise des enseignants requiert une revalorisation statutaire et une clarification de leurs fonctions. Leur autorité ne peut résulter seulement d'un travail sur la relation pédagogique, sur leurs compétences didactiques et leurs pratiques de la sanction et de la loi (qui restent néanmoins à initier) mais en appelle fondamentalement à une analyse de la précarité actuelle de leur situation à la lumière des évolutions historiques de la fonction. Ces questions doivent être mises en mouvement par un travail scientifique rigoureux. Mais ces données manquent. Les entretiens confirment une difficulté à mobiliser une réflexion collective sur les pratiques passées et une volonté de ne pas y revenir. Les réticences existent sur le terrain mais aussi parmi les chercheurs sur ce sujet sensible (Prucha, 2002, Spilková, 2005).

\section{Conclusion}

A l'issue de la recherche, si nous voulons requalifier la relation d'autorité en République tchèque, il convient d'abord de se démarquer d'une interprétation courante, de défection généralisée et inéluctable de la fonction paternelle qui comprend la violence comme un produit de la décadence moderne, liée à la dévaluation d'un pouvoir autoritariste (Debarbieux, 2006). Le contexte spécifique d'un pays post communiste nous apprend que cette prise de distance doit être articulée à une description et à une analyse précises des rapports complexes des acteurs à l'histoire, sans que le passé ne soit considéré a priori comme un poids, un obstacle ou au contraire une simple parenthèse sans effet. Les évolutions sont complexes, quelquefois paradoxales, irréductibles aux simplismes de la décadence, de l'autoritarisme ou d'une conversion simple et soudaine à des valeurs démocratiques mal définies. 
L'école tchèque se débat avec l'injonction d'une rupture visible avec des pratiques pédagogiques plutôt magistrales et dogmatiques (Spilková, 2005), orientées plutôt vers le conformisme. La démocratie requiert des pratiques discursives et institutionnelles différentes, témoignant de l'exercice libre d'une parole critique, responsable et constructive par les (futurs) citoyens que sont les élèves et les enseignants. Sous les apparences d'une rupture avec le passé (les élèves se disent irrespectueux à l'égard des enseignants), parfois caricaturé (la répression exercée n'était pas grossière, homogène et purement externe aux sujets), une certaine continuité apparaît. C'est celle, par exemple, d'une autocritique (« impolitesse » auto déclarée des élèves) qui a pour fonction essentielle la perpétuation des normes collectives en vigueur (conformité aux normes enseignantes, « politesse » comme critère le plus pertinent). C'est paradoxalement en adoptant les normes et les terminologies de leurs aînés que les élèves commencent à exprimer leur rejet à cet égard. Une mutation semble émerger mais parallèlement, les élèves et les enseignants restent liés par une représentation stéréotypée de l'autorité (Spilková, 2005) à l'égard de laquelle la société de la transformation est ambivalente.

La difficulté fondamentale de la relation pédagogique dans le contexte tchèque semble consister dans un malentendu sur ce que signifie la singularité subjective et l'autorité comme fait, pour un sujet, de «se faire soi-même son propre auteur » (Ardoino, 2000, p. 200). Dans le contexte des postcommunismes où les sphères privées et publiques, individuelles et collectives, administratives et politiques ont été volontairement brouillées par le socialisme, leurs frontières se réagencent (Kott, Mespoulet, 2006), posant actuellement de manière nouvelle les questions du rapport à l'autre et de la construction subjective. Celles ci ne peuvent exister sans une loi qui marque les limites et les différences, une loi qui vise l'éducation et non l'obéissance, la sanction et non la répression, la critique constructive et non seulement la politesse formelle. L'enquête montre les conséquences problématiques d'une pédagogie visant la stricte conformation des comportements c'est-à-dire uniquement l'obéissance. L'autorité est essentiellement fondée sur une confiance en l'autre qui se construit en amont des actes répressifs. A cet égard, le « tout sécuritaire » est tout autant une fiction destructrice qu'une démocratie où la sanction n'existerait pas. C'est un autre danger qui guette les systèmes éducatifs postcommunistes, reposant sur un discours d'ostalgie (forme de regret propre aux anciens pays de l'Est, Blaive, 2006) et de décadence libérale.

Magdalena Kohout-Diaz, ERCEF, équipe de recherche comparative en éducation et formation, Observatoire international de la violence scolaire, IUFM d'Aquitaine - université Montesquieu Bordeaux 4 \& université Victor-Segalen Bordeaux 2.

Eric Debarbieux, directeur de l'ERCEF, équipe de recherche comparative en éducation et formation, directeur de L'Observatoire international de la violence à l'école, université Victor-Segalen Bordeaux 2. 


\section{BIBLIOGRAPHIE}

Arendt H. (1968). Qu'est-ce que l'autorité? In La crise de la culture. Paris: Gallimard, 1972.

Ardoino J. (2000). Les avatars de l'éducation, Paris: PUF.

Benbenisthy R. \& Astor R.A. (2006). School Violence in Context: Culture, Neighborhood, Family, School and Gender. New York: Oxford University Press.

Bendl S. (2004). Kázen`ské problémy ve škole. [Les problèmes d’autorité à l'école.] Praha: Triton.

Blaive M. (2006). Nostalgie et mémoire collective du communisme en République tchèque. Les enseignements d'une enquête. In Kott, S., Mespoulet, M., Eds. (2006). Le postcommunisme dans l'histoire. Bruxelles: Éditions de l'université de Bruxelles.

Blaya C. (2006). Violences et maltraitances en milieu scolaire. Paris: Colin.

Caumont F. (2006). La question de l'autorité au regard de la fonction paternelle. In Dialogue, $n^{\circ} 173,2006-3$, p. 113-124.

Debarbieux E. (1996). La violence en milieu scolaire. T. 1 État des lieux. Paris: ESF.

Debarbieux E. (2003). Les micro violences et le climat scolaire: évolution 1995-2003 en écoles et en collèges. Recherche commanditée par le conseil régional d'Aquitaine et le ministère de l'Éducation nationale (DESCO2) - MEN.

Debarbieux E. (2006). Violence à lécole: un défi mondial? Paris: Colin.

Devaux S. Savoir et pouvoir politique. Le rôle des scientifiques dans la constitution des ministères de l'Environnement en Tchécoslovaquie (1989-1992). In Kott S., Mespoulet M., eds. (2006). Le postcommunisme dans l'histoire. Bruxelles: Éditions de l'université de Bruxelles.

Gottfredson D.C. (2001). Schools and delinquency, Cambridge: University Press.

Greger D. (2005). Développement et débat autour du modèle de collège unique en République tchèque et dans d'autres pays d'Europe centrale et orientale. Communication présentée au colloque « Construction/ déconstruction du collège unique », université de Paris VIII (Saint-Denis), 28 octobre 2005.

Havlínová M., Kolár, M. (2001). Sociální klima v prostredí základních škol Ceské republiky. [Climat social dans les écoles élémentaires de la République tchèque]. Rapport au ministère de l'Éducation et des Sports tchèque. Praha: MŠMT CR.

Hawker D. S. J., Boulton M. J. (2000). Twenty years research on peer victimization and psychosocial maladjustment: A meta-analytic review of cross-sectional studies. Journal of Child Psychiatry and Psychiatry, 41, 441-455.

Kohout-Diaz M. (2006). Violence scolaire en République tchèque: šikana? Thèse de doctorat $n^{\circ} 1360$, mention sciences de l'éducation, Bordeaux, université V. Segalen, Bordeaux 2, 446 p.

Kott S., Mespoulet M. (eds.) (2006). Le postcommunisme dans l'histoire. Bruxelles: Éditions de l'université de Bruxelles.

LacadéE P., (2003). Le malentendu de l'enfant. Dijon: Payot Lausanne-Nadir.

Olweus D. (1993). Bullying at school: what we know and what we can do. Oxford: Blackwell.

PARízer V. (2000). Sociální a ekonomické postavení ucitelu, jeho príciny a cesty ke zlepšení. [La situation sociale et économique des enseignants, ses causes et les voies de son amélioration.] In Promeny školy, ucitele a žáka na prelomu tisiciletí. Protokol ze sjezdu ucitelu v Brne 30.-31. srpna 2000. [Les transformations de l'école, de l'enseignant et de l'élève au tournant du millénaire. Actes du rassemblement des enseignants à Brno le 30.-31. septembre 2000.] Konvoj, spol. sr.o., Brno 2001. p. 73-74. 
Prairat, E. «Questions premières ». In Prairat, E. (Ed.) La sanction. Approches plurielles. Cahiers A. Binet. n 668, 2001, n 3. Paris: Erès. p. 75-86.

Prairat, E. Du respect et de l'hospitalité. In Prairat E., Andrieu B. (Eds.) Les valeurs: savoir et éducation à l'école. Nancy: Presses universitaires de Nancy, 2003, p. 105-116.

Prairat, E. De la déontologie enseignante. Paris: PUF, 2005.

Prokop, J. « Kritika a mýty soucasné školy ». [Critique et mythes de l'école actuelle.] In Chráska M., Tomanová D., Holoušová D. (Ed.) Klima soucasné ceské školy. [Le climat de l'école tchèque actuelle, Actes de la onzième conférence de la Société pédagogique tchèque.] Brno: Konvoj, 2003, p. 64 -63.

Prucha, J. Ucitel. Soucasné poznatky o profesi. [L'enseignant: connaissances actuelles sur la profession.] Prague: Portál, 2002.

Prucha, J. Czech Republic. In Hörner W. et al. (Eds.). The Education Systems of Europe. Dortrecht: Springer, 2007. p. 166-183.

RAGARU, N. «Quelques remarques sur les échanges de services et l'appropriation de l'ordre politique en Bulgarie communiste ». In Kott, S., Mespoulet, M., Eds. (2006). Le postcommunisme dans l'histoire. Bruxelles: Éditions de l'université de Bruxelles, 2006.

Reboul, O. La philosophie de l'éducation. Paris: PUF, 1989.

Smith P.K., Morita Y., Junger-Tas J., Olweus D., Catalano \& Slee P. (ed.). The Nature of school bullying, a cross-national perspective, London: Routledge, 1999.

SPILKOVÁ, V. « Les tentatives de mise en place des "pédagogies nouvelles " dans les écoles fondamentales tchèques après 1989 ». Revue française de pédagogie, n 153, octobre-novembre-décembre 2005, p. 25-38 (traduit du tchèque par M. Springer avec la collaboration de la rédaction). 
Claude Carpentier (coord.). L'école dans un monde en crise. Entre globalisation et héritages. L'Harmattan, 2008, (Éducation et société), 284 p. ISBN : 978-2-296-05089-1 (25,65€).

es crises traversées par l'institution scolaire partout dans le monde se situent au point de convergence entre « modernité » dominée par le néolibéralisme et héritages historiques. La première a pour conséquence l'émergence d'un paradigme selon lequel l'éducation devient un produit soumis aux règles des échanges économiques et de la concurrence. Ce qui rend obsolète le principe d'une éducation considérée comme bien public au service de la réduction des inégalités.

Aboutissement du processus de développement du capitalisme, la crise actuelle de l'école témoigne également de l'héritage des bouleversements historiques liés à la décolonisation et aux nouveaux rapports «nord-sud » ainsi qu'à la conversion des sociétés " socialistes » au néolibéralisme. Dans les deux cas, la question des identités culturelles et ethniques est au cœur de la crise des institutions scolaires.

C'est dans ce contexte que les enseignants, acteurs privilégiés des crises de l'école, se trouvent confrontés à une expérience sociale traversée par les tensions, aussi bien en ce qui concerne leur identité professionnelle mise à rude épreuve par la transformation de l'institution qu'en ce qui concerne l'évolution des contenus d'enseignement eux-mêmes.

La coordination de l'ouvrage et la présentation des textes ont été assurées par des membres du CURSEP (Centre universitaire de recherche en sciences de l'éducation et en psychologie, équipe d'accueil 2089) : Claude Carpentier, directeur. Bruno Poucet, Teresa Longo, Philippe Monchaux, Yves Verneuil. 